\title{
Terapia de la resincronización cardiaca transvenosa en la población pediátrica
}

\author{
Diego I. Vanegas*, Carlos A. Rincón y David Santacruz \\ Unidad de Electrofisiología Cardiovascular, Hospital Militar Central, Bogotá, Colombia
}

Recibido el 10 de noviembre de 2015; aceptado el 21 de enero de 2016

Disponible en Internet el 2 de marzo de 2016

\author{
PALABRAS CLAVE \\ Cardiopatía \\ congénita; \\ Bloqueo cardiaco; \\ Marcapasos; \\ Asincronía; \\ Falla Cardiaca
}

\section{KEYWORDS}

Congenital hear

disease;

Cardiac block;

Pacemaker;

Asynchrony;

Cardiac failure

\begin{abstract}
Resumen La disfunción ventricular izquierda y la cardiopatía dilatada (CD), debidas a la estimulación crónica ventricular derecha son complicaciones reconocidas en adultos y niños. La terapia de la resincronización cardiaca (TRC), ha demostrado beneficios en los adultos con disfunción ventricular izquierda $(\mathrm{FEVI}<35 \%)$, deterioro de la clase funcional y bloqueo de la rama izquierda (intrínseco o inducido por estimulación). En los niños la incidencia de cardiomiopatía dilatada es desconocida y no existen guías o grandes estudios sobre la resincronización cardiaca, por lo que no es posible extrapolar las recomendaciones de los pacientes adultos. Se presenta la experiencia en una paciente con bloqueo $\mathrm{A}-\mathrm{V}$ completo postquirúrgico tras corrección de cardiopatía congénita, portadora de un marcapaso quien desarrolló la falla cardiaca, esta fue tratada exitosamente mediante la resincronización biventricular en dos oportunidades, con recuperación de la clase funcional y los parámetros hemodinámicos.

(c) 2016 Sociedad Colombiana de Cardiología y Cirugía Cardiovascular. Publicado por Elsevier España, S.L.U. Este es un artículo Open Access bajo la licencia CC BY-NC-ND (http:// creativecommons.org/licenses/by-nc-nd/4.0/).
\end{abstract}

\section{Transvenous cardiac resynchronization therapy in pediatric population}

Abstract Left ventricular dysfunction and dilated cardiomyopathy (DC) caused by right ventricular chronic stimulation are recognised complications in adults and children. Cardiac resynchronization therapy (CRT) has shown benefits in adults with left ventricular dysfunction (LVEF $<35 \%$ ), deterioration of functional class and left bundle-branch block (instrinsic or induced by stimulation). Incidence of dilated cardiomyopathy in children is unknown, and there are no guides or big studies about cardiac resynchronization therapy, so recommendations for adult patients cannot be extrapolated. The experience of a female patient with a pacemaker

\footnotetext{
* Autor para correspondencia.

Correo electrónico: diegovanegascadavid@gmail.com (D.I. Vanegas).
} 
and postoperative complete AV block after correction of a congenital heart disease is presented; she developed heart failure that was successfully treated with biventricular resynchronization in two opportunities, with recovery of functional class and hemodynamic parametres.

(c) 2016 Sociedad Colombiana de Cardiología y Cirugía Cardiovascular. Published by Elsevier España, S.L.U. This is an open access article under the CC BY-NC-ND license (http:// creativecommons.org/licenses/by-nc-nd/4.0/).

\section{Introducción}

La epidemiología de la falla cardiaca en los niños es más amplia que en los adultos, destacándose las cardiopatías congénitas que en los menores de 19 años de edad, corregidas o no quirúrgicamente, son responsables del 61 a $82 \%$ de los casos de insuficiencia cardiaca. En las miocardiopatías la incidencia es de 1.13 casos anuales por cada $100 \mathrm{mil}$ niños, siendo más frecuente la cardiomiopatía dilatada con mortalidad de $13,6 \%$ a dos años ${ }^{1}$.

En los niños la prevalencia e incidencia de la cardiopatía dilatada secundaria al uso de estimulación ventricular derecha por bloqueo aurículo-ventricular completo es desconocida $^{2-4}$. Entre los mecanismos se postulan la estimulación ventricular derecha que induce remodelamiento del ventrículo izquierdo, el alto porcentaje de estimulación y la necesidad de estimular a frecuencia cardiaca elevada debido a la edad del paciente p- $^{5-8}$.

La estimulación cardiaca en pediatría se realiza mediante los electrodos epicárdicos insertados por toracotomía aunque también se práctica vía transvenosa subclavia. A favor de esta última estrategia se encuentran las dificultades técnicas para acceder al pericardio por la presencia de adherencias y cicatrices cuando el paciente ha sido previamente intervenido, los umbrales altos de detección y la estimulación epicárdica y el riesgo incrementado de infección cuando se requiere de una reintervención.

La aproximación transvenosa también tiene limitaciones: el tamaño del paciente, el diámetro de la vena subclavia y del seno coronario, el progresivo desarrollo pondo-estatural que puede llevar al desalojo y/o disfunción de los electrodos y la escasa experiencia del operador en esta población.

Se presenta el caso de una paciente con bloqueo aurículo-ventricular completo postquirúrgico, portadora de marcapaso, quien desarrolló la falla cardiaca, fue tratada exitosamente con terapia de resincronización cardiaca biventricular transvenosa en dos oportunidades, con mejoría de la clase funcional y de los parámetros hemodinámicos.

\section{Reporte de caso}

Un paciente de sexo femenino quien a los 11 meses de edad, requirió de implante vía subclavia izquierda de marcapasos unicameral ventricular derecho por bloqueo aurículo-ventricular completo en el postoperatorio de cierre a tórax abierto de una comunicación interventricular. Ocho años después la paciente presentó síntomas de la falla cardiaca a pesar del manejo médico apropiado con inhibidores de la ECA, el diurético y la digoxina.

El electrocardiograma mostró ritmo de marcapasos vvı con captura ventricular al 100\%, QRS ancho de $135 \mathrm{mseg}$ y morfología del bloqueo de rama izquierda. La impedancia había aumentado 150 Ohms en relación a la evaluación precedente. La radiografía de tórax documentó: índice cardiotorácico mayor a 0,5 a expensas del ventrículo izquierdo, sin signos de hipertensión pulmonar pre o postcapilar ni derrame pleural. El electrodo del marcapasos había perdido asa observándose tenso con su extremo distal localizado en el tercio medio del ventrículo derecho.

El ecocardiograma transtorácico confirmó un severo compromiso de la función sistólica y diastólica ventricular izquierda (FEVI: 18\%), dilatación ventricular izquierda (VFsVI $108 \mathrm{~mL}$, VFdVI: $132 \mathrm{~mL}$ ). No se observó corto circuito residual. Se documentó movimiento paradójico del septum.

Se realizaron las siguientes consideraciones: 1) Reacomodar el electrodo ventricular derecho suponía extrema dificultad técnica por el tiempo trascurrido desde el implante. De igual forma, implantar un electrodo nuevo al ventrículo derecho no mejoraría la disfunción contráctil. 2) El implante de un electrodo al ventrículo izquierdo, epicárdico o transvenoso se hacía mandatorio para intentar un remodelamiento reverso. 3) Al tiempo, de ser posible y para lograr una estimulación biventricular se haría necesario implantar otro electrodo ventricular derecho transvenoso.

\section{Descripción del procedimiento}

Bajo anestesia general se realizaron los siguientes pasos:

1) Se canalizó la vena femoral derecha y se introdujo un electrocatéter decapolar deflectable $5 \mathrm{Fr}$ al seno coronario realizando la evaluación primaria de su tamaño y curso.

2) Se realizó venografía de la yugular interna, subclavia izquierda y cava superior. Se demostró permeabilidad y tamaño de la subclavia izquierda ocupada parcialmente por un electrodo.

3) Se practicó incisión de la región deltopectoral izquierda sobre el marcapasos y se extrajó. Se desconectó el electrodo disfuncionante y se disecó hasta la clavícula. Se observó pérdida del aislamiento del electrodo bajo la clavícula con imposibilidad de avanzar un estilete. Dada la pérdida del aislamiento se cortó y abandonó para la extracción femoral.

4) Se realizó punción subclavia izquierda dilatando sucesivamente hasta lograr avanzar un sistema "peel away" 7 Fr por donde se introdujó el electrodo del seno coronario.

5) Usando un estilete de consistencia suave de curva preformada y en proyección oblicua izquierda se logró introducir el electrodo en la porción inicial del seno 
coronario. No se utilizó el sistema de canalización usado en los adultos.

6) Se retiró el estilete y se cambió por una guía extrasoporte la cual se avanzó "a ciegas"' a través del seno coronario sin utilizar la venografía y se posicionó en la región lateral y anterior del ventrículo izquierdo. Se avanzó luego el electrodo hasta su máxima resistencia. Se verificó que existieran detección y captura adecuadas; se dejó adecuada asa en la aurícula derecha y se fijó al músculo pectoral.

7) Dada la limitación de espacio en la vena subclavia por alojamiento de dos electrodos y para reemplazar el electrodo ventricular derecho disfuncionante, se puncionó la vena yugular izquierda. Se avanzó un sistema "peel away" 7Fr y a través del mismo se introdujó un electrodo de fijación activa al VD. Tras verificar adecuados umbrales se fijó el electrodo al músculo platisma del cuello, protegiendo el tambor con algunos haces musculares.

8) Utilizando un tunelizador se disecó el tejido celular subcutáneo hasta alcanzar la región deltopectoral izquierda y se pasó por esa vía el electrodo ventricular derecho hasta el bolsillo conectándose junto al electrodo del seno coronario al dispositivo de la resincronización cardiaca; se obturó el canal atrial y se fijó el generador al plano prepectoral izquierdo.

\section{Seguimiento}

Se dejó la estimulación simultánea de los dos ventrículos obteniéndose un QRS de 100 mseg (Fig. 1). Al mes se documentó por la ecocardiografía remodelamiento reverso con mejoría sustancial y temprana de la FEVI de 18 a 43\%, (Fig. 2). La clase funcional pasó de III a I, condición clínica en la que se mantuvo durante los siguientes 3 años. A la edad de 11 años, la paciente fue llevada al servicio de urgencias por historia de 20 días de evolución de pobre tolerancia al ejercicio, deterioro de su clase funcional hasta III-IV, ortopnea, oliguria y edema de miembros inferiores. Se identificó ingurgitación yugular grado ।-Il, crépitos bibasales y edema grado । de miembros inferiores. Se manejó en la unidad de cuidados intensivos pediátrica con soporte inotrópico y tratamiento farmacológico con pobre respuesta. $\mathrm{El}$ nuevo ecocardiograma informó compromiso de la FEVI (22\%); la radiografía de tórax demostró pérdida del asa y desalojo del electrodo del seno coronario. Se identificó la estimulación diafragmática al aumentar el voltaje de salida del canal ventricular izquierdo. La detección y captura del electrodo ventricular derecho era óptima. Por la ausencia de mejoría, la paciente se consideró posible candidata a trasplante cardiaco. Dadas las anteriores circunstancias se ofreció nuevamente resincronización inicialmente transvenosa y de no ser posible epicárdica. El segundo procedimiento de resincronización biventricular transvenosa tuvo pasos similares a los descritos arriba siendo relevante:

1) Intento fallido de reposicionar el electrodo del seno coronario. Se intentó avanzar un estilete a su través pero solo se logró hasta el segmento medio. Mediante delicada tracción manual se desprendió del seno coronario pero su extracción fue imposible por retención en la vena cava superior.
2) Utilizando un dispositivo de extracción transfemoral (Asa Needle's Eye Snare), se capturó el extremo distal del cable del seno coronario. Se cortó el extremo proximal del mismo, bajo la clavícula y se extrajó vía femoral.

3) Se realizó punción subclavia izquierda y utilizando la misma técnica del primer procedimiento se canuló el seno coronario y se insertó el electrodo posicionándolo en la región anterolateral del ventrículo izquierdo. Se diseñó un asa amplia con el electrodo en la aurícula derecha. Se conectaron los electrodos del ventrículo derecho y del seno coronario a sus respectivos canales en un nuevo dispositivo de la resincronización cardiaca (Fig. 3).

Al tercer día postquirúrgico se realizó el ecocardiograma transtorácico identificándose un nuevo incremento en la FEVI a 35\%. La paciente fue dada de alta al quinto día postoperatorio en ausencia de síntomas de la falla cardiaca permaneciendo en clase funcional । durante el primer trimestre de seguimiento.

\section{Discusión}

La estimulación biventricular transvenosa en más de una ocasión (a los 8 y 11 años de edad), y en una misma paciente es lo más relevante de este caso. Como esta niña, otros pacientes pediátricos con cardiopatía dilatada secundaria a estimulación ventricular derecha por marcapasos pueden ser dados de alta de una lista de espera para trasplante cardiaco dado que son altamente "respondedores" a la terapia de la resincronización cardiaca ${ }^{11-15}$.

Una de las limitaciones al uso de la terapia de la resincronización biventricular transvenosa en la población pediátrica es la potencial dificultad técnica de acceso vascular subclavio y/o del seno coronario dado el tamaño corporal y/o el bajo peso del paciente. No obstante, es posible estimar la posibilidad de éxito mediante la medición por venografía de la vena subclavia y el uso en determinadas circunstancias de la vena yugular interna ipsilateral. Así mismo, el tamaño, el curso y los vasos tributarios del seno coronario pueden evaluarse mediante su canalización vía femoral utilizando los electrocatéteres 4, 5 o 6 Fr.

En la población pediátrica con cardiopatía dilatada inducida por estimulación ventricular derecha existen otros factores a tener en cuenta en relación a la terapia de la resincronización cardiaca como alternativa de manejo: 1) Es necesario confirmar que no existe una causa mecánica residual corregible. 2) En la valoración preoperatoria la demostración de disincronía intra e interventricular mediante la ecocardiografía y el registro de QRS ancho ajustado para la edad del paciente. 3) La decisión médica de intentar la terapia de la resincronización cardiaca transvenosa vs. tórax abierto, también depende en gran medida de la experiencia del operador.

Además, del tamaño de los vasos, otra objeción al acceso transvenoso ha sido el hecho demostrado de la pérdida de "asa" del electrodo o electrodos endocárdicos que ocurre por el crecimiento en la talla y el peso del paciente. Lo anterior lleva a la disfunción del mismo y con los años a la pérdida de captura. No obstante, si se toma la precaución durante el primoimplante de dejar el electrodo con un asa flotante lo suficientemente holgada, 
1: I $2,5 \mathrm{~mm} / \mathrm{mV}$
2: II $5,0 \mathrm{~mm} / \mathrm{mV}$

3: Marcadores
4: Punta unipolar A Ganancia auto $(3,7 \mathrm{~mm} / \mathrm{mV})$

5: Ampl. Detección V Ganancia auto $(1,4 \mathrm{~mm} / \mathrm{mV})$

Velocidad de barrido: $25 \mathrm{~mm} / \mathrm{s}$

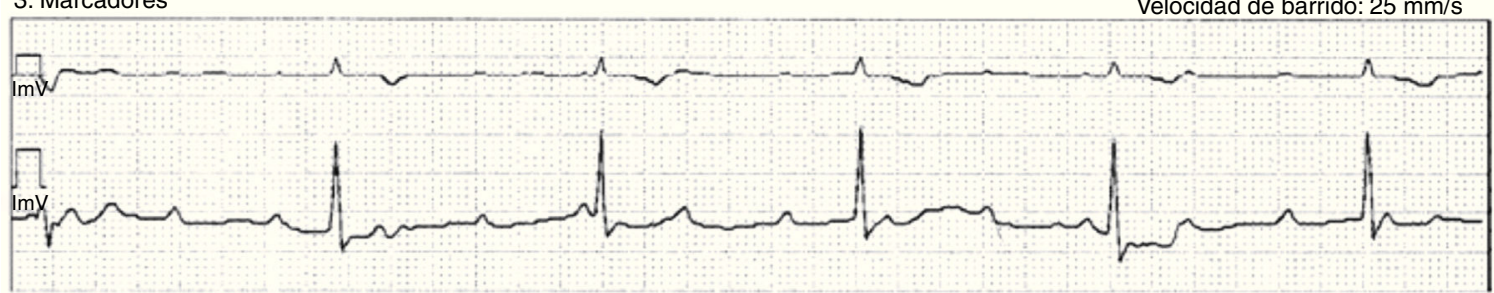

$1: 12,5 \mathrm{~mm} / \mathrm{mV}$

2: II $5,0 \mathrm{~mm} / \mathrm{mV}$

4: Punta unipolar A Ganancia auto $(3,7 \mathrm{~mm} / \mathrm{mV})$

3: Marcadores

5: Punta unipolar VD Ganancia auto $(1,4 \mathrm{~mm} / \mathrm{mV})$

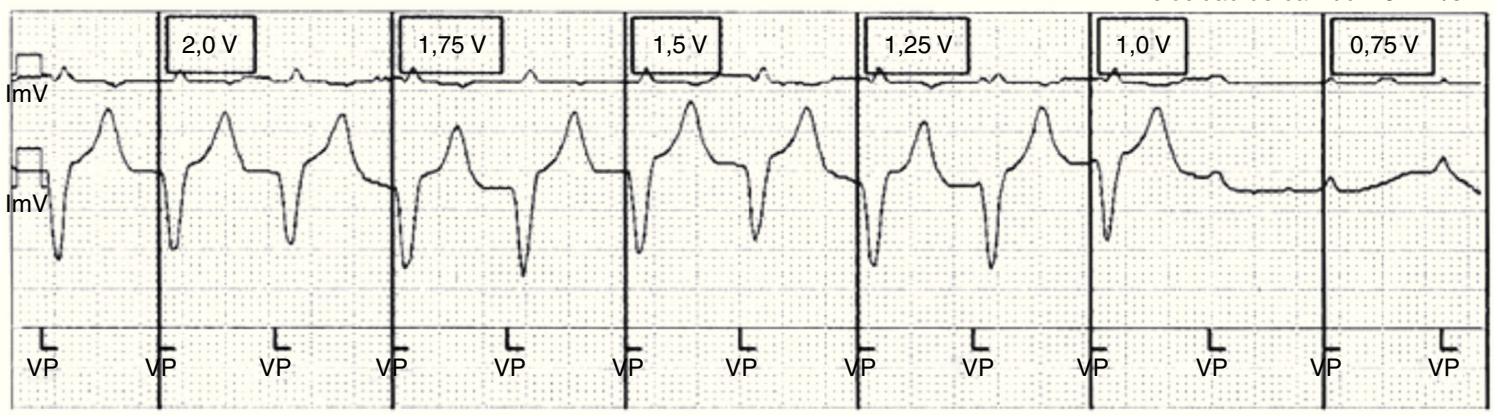

1: $12,5 \mathrm{~mm} / \mathrm{mV}$

2: II $5,0 \mathrm{~mm} / \mathrm{mV}$

4: Punta unipolar A Ganancia auto $(3,7 \mathrm{~mm} / \mathrm{mV})$

3: Marcadores

5: Punta unipolar VD Ganancia auto $(1,4 \mathrm{~mm} / \mathrm{mV})$

Velocidad de barrido: $25 \mathrm{~mm} / \mathrm{s}$
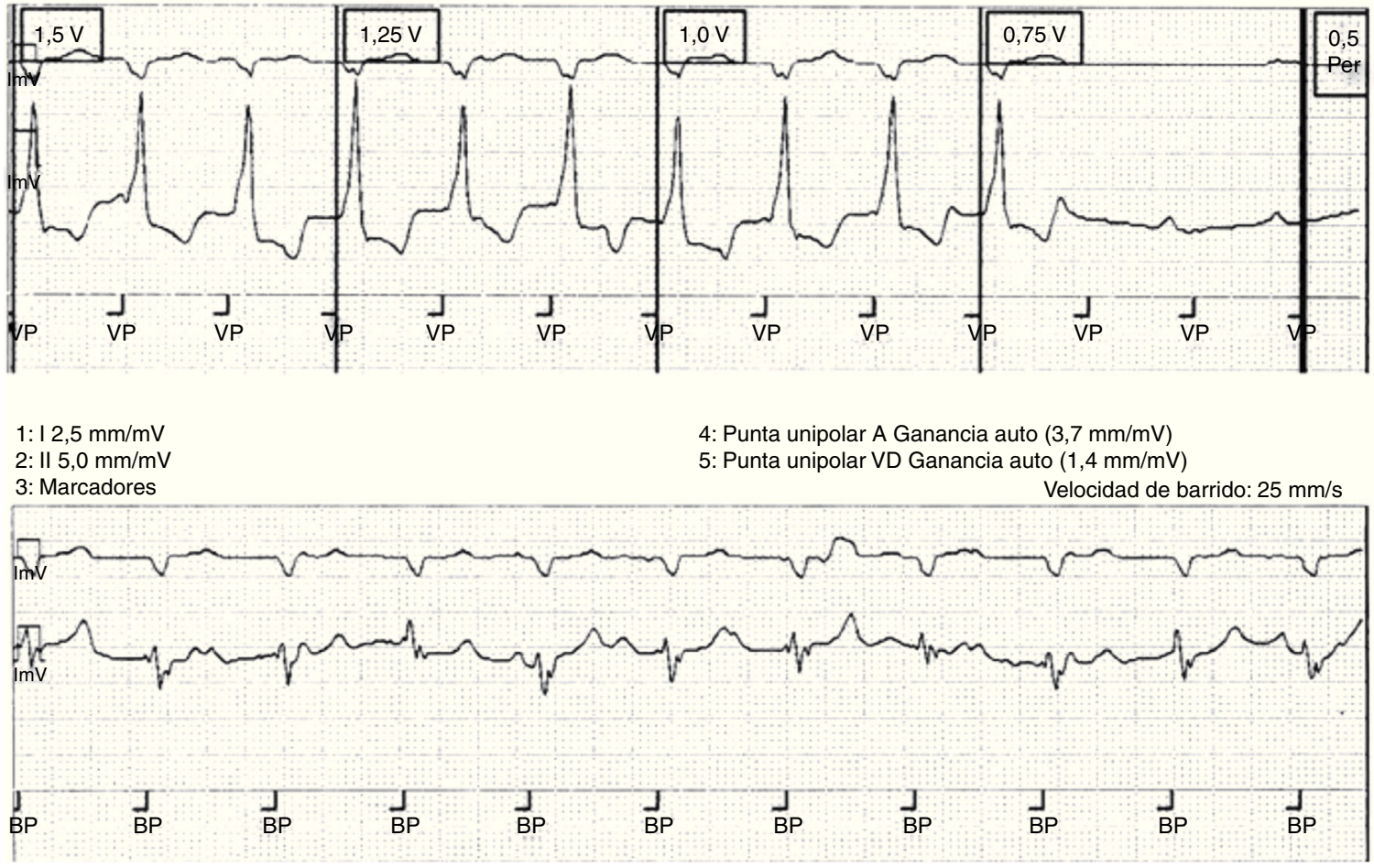

Figura 1 Trazos electrocardiográficos en dos derivaciones: । - ॥; velocidad $25 \mathrm{~mm} / \mathrm{seg}$, amplitud primer canal $2,5 \mathrm{~mm} / 1 \mathrm{mv}$ y segundo canal $5 \mathrm{~mm} / 1 \mathrm{mv}$. De arriba hacia abajo: ritmo intrínseco con bloqueo A-v completo y escape unional a 35 lpm; ritmo de estimulación ventricular derecha, QRS ancho de $120 \mathrm{mseg}$; ritmo de estimulación ventricular izquierda, QRS ancho de 120 mseg; ritmo de estimulación biventricular, QRS estrecho 100 mseg de duración. 


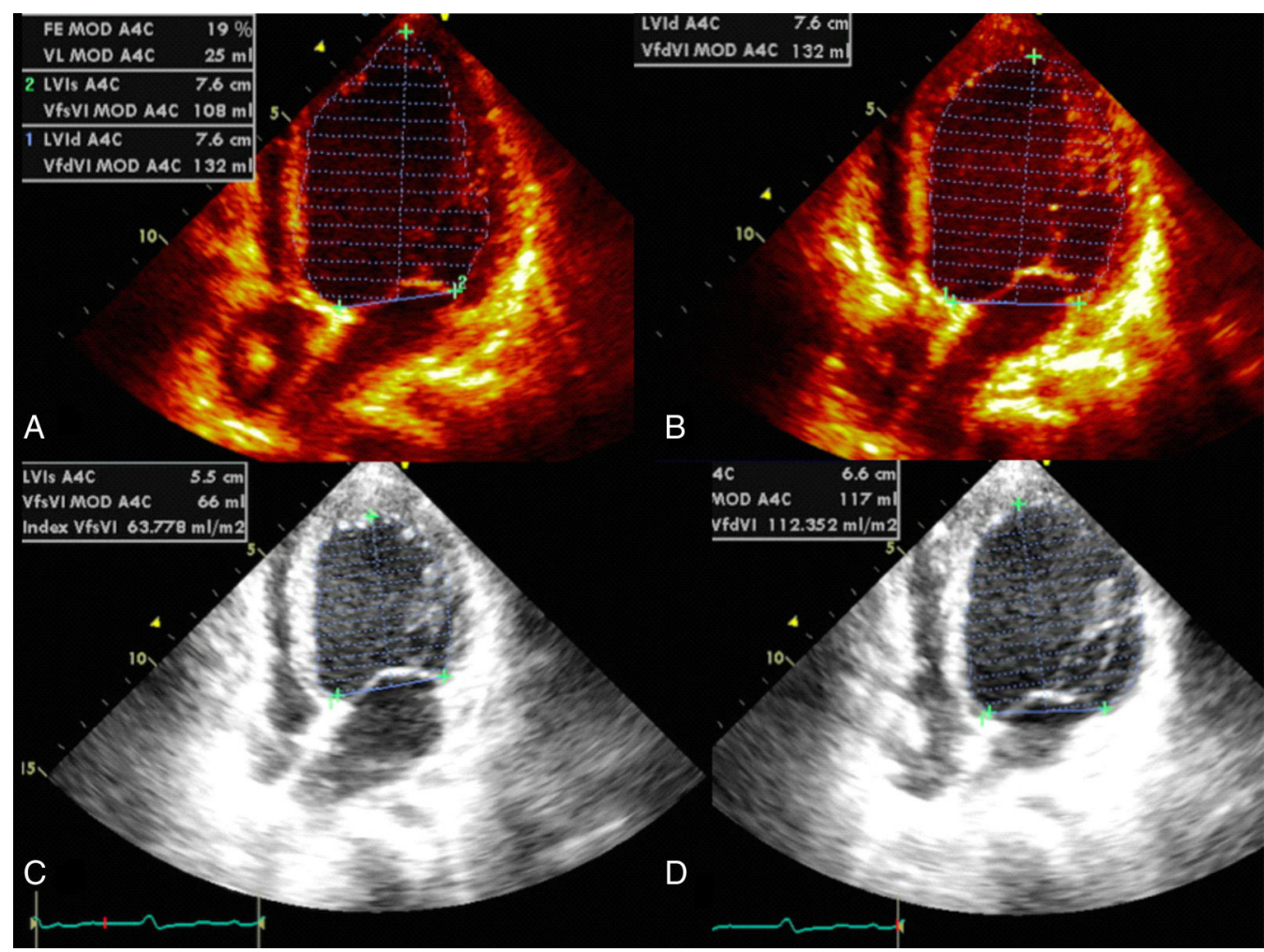

Figura 2 Valoración ecocardiográfica preoperatoria (A-B) y postoperatoria (C-D); se aprecia cambio significativo en los volúmenes de fin de diástole y de sístole y por consiguiente de la fracción de eyección, pasando de 18 a $43 \%$. A) VfsVI A4C 108 ml. B) VfdVI $132 \mathrm{ml}$. FEVI: 18\%, por Simpson biplano. Posterior a la resincronización: C) VfsVI A4C $66 \mathrm{ml}$. D) VfdVI A4C 112 ml, FEVI 43\% por Simpson biplano. VfsVI (Volumen de fin de sístole ventrículo izquierdo), VfdVI (volumen de fin de diástole ventrículo izquierdo).
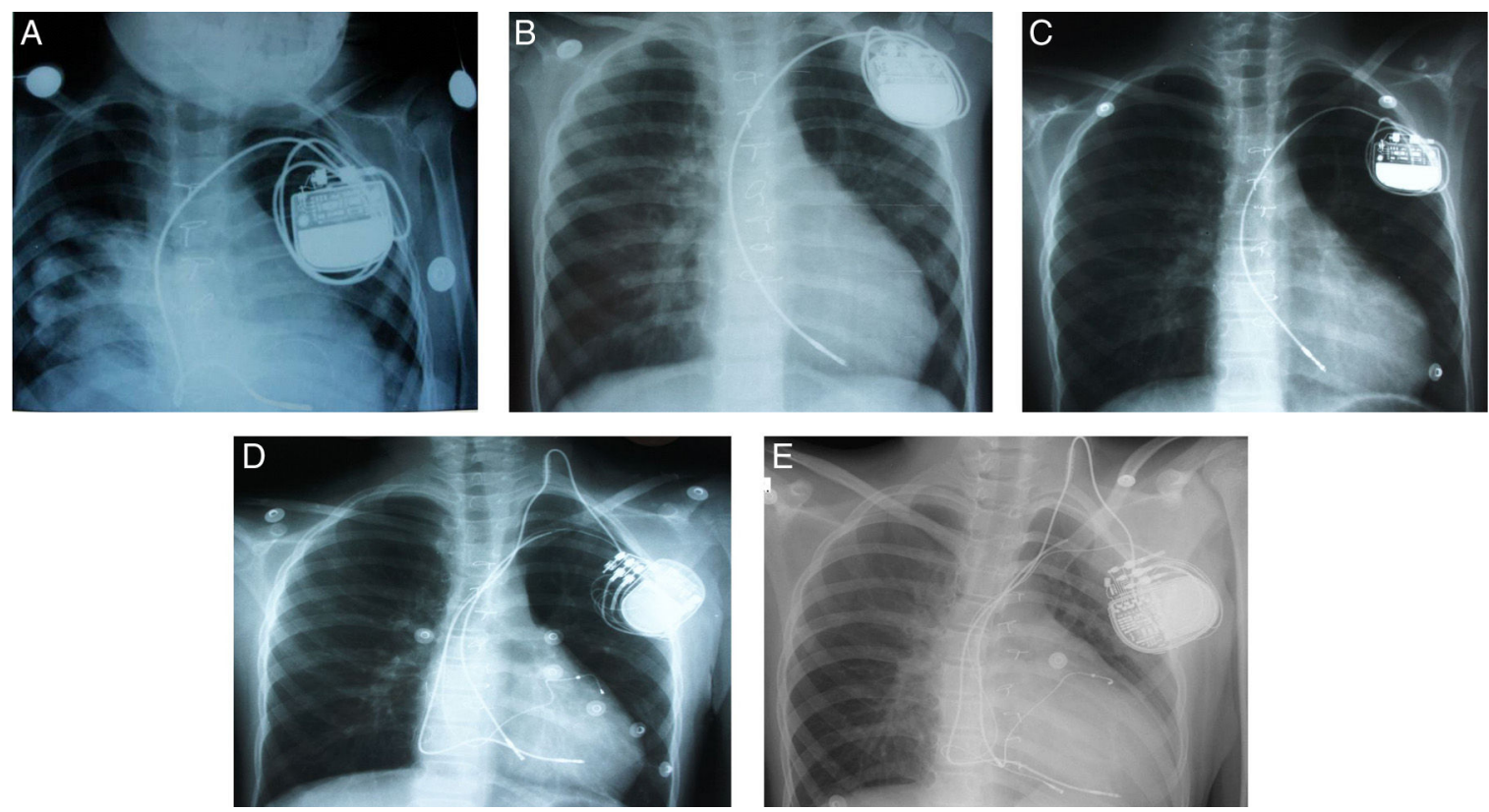

Figura 3 Seguimiento radiológico: A) A los 11 meses de edad, marcapaso unicameral vvi-R asa de electrodo visible en la parte baja de la imagen. B) A los 3 años de edad. C) A los 8 años de edad, el electrodo ventricular derecho en su máxima elongación con incremento anormal de la impedancia. D) Tras el implante de la resincronización biventricular, el electrodo ventricular derecho vía yugular y el seno coronario vía subclavia izquierda; se observa el electrodo disfuncionante cortado y abandonado. E) A los 12 años de edad, tras la segunda intervención motivada por el desalojo del electrodo del seno coronario. 
la disfunción predecible del mismo por el crecimiento del paciente puede tardar años en presentarse.

El minucioso seguimiento clínico, radiográfico y por telemetría pueden indicar cuando es el mejor momento para la inserción de uno o dos nuevos electrodos, derecho y/o izquierdo, con o sin extracción del primero de acuerdo a la condición clínica del paciente. Para ese momento, el tamaño del vaso subclavio y/o del seno coronario ha aumentado en forma paralela al crecimiento del niño, posiblemente, lo suficiente para alojar otro u otros electrodos. El acceso venoso yugular interno ipsilateral es una alternativa cuando el espacio para introducir más de un electrodo transvenoso es limitado?.

En los pacientes adultos la TRC ha demostrado que mejora la hemodinámica cardiaca, la FEVI y los síntomas de falla al tiempo que incrementa la tolerancia al ejercicio y la supervivencia ${ }^{10}$. En niños, la resincronización ha sido utilizada en diversos escenarios, así: 1) Estimulación biventricular o ventricular izquierda en pacientes con estimulación bicameral atrioventricular crónica que han desarrollado la falla cardiaca. 2) Estimulación biventricular epicárdica con liberación exitosa de la circulación extracorpórea después de la cirugía correctiva de la enfermedad cardiaca congénita. 3) Pacientes con ciertas formas de cardiopatías congénitas asociadas con la falla ventricular y, 4) En cardiomiopatía dilatada idiopática ${ }^{11,12}$.

Strieper y Cols., describieron los efectos benéficos clínicos de la TRC en una serie de casos de pacientes con la cardiopatía congénita considerados para trasplante cardiaco, la mayoría retirados de la lista de espera debido a mejoría sintomática. Aproximadamente, el $60 \%$ de los pacientes reportados y que cursaron con mejoría clínica habían recibido marcapasos ventricular derecho por bloqueo aurículo-ventricular completo congénito ${ }^{13}$.

En un estudio multicéntrico que incluyó 22 instituciones, los autores evaluaron la eficacia y seguridad a corto plazo de la terapia de resincronización cardiaca en 103 niños con falla cardiaca congestiva, demostrando mejoría de la función ventricular izquierda y acortamiento de la duración del complejo QRS ${ }^{14}$. Moak y Cols., describieron 6 pacientes que desarrollaron insuficiencia cardiaca y cardiopatía dilatada posterior al implante de marcapasos ventricular derecho por bloqueo aurículo-ventricular completo congénito que posteriormente fueron llevados a la resincronización cardiaca, demostrando beneficios en la función cardiaca y otros desenlaces clínicos. Ellos sugieren con base en sus resultados realizar la actualización temprana a sistemas de estimulación biventricular en pacientes portadores de marcapasos ventricular derecho y cardiopatía dilatada ${ }^{15}$.

Para la utilización de la terapia de la resincronización cardiaca en pediatría se debe considerar que ciertos criterios utilizados en los adultos no aplican estrictamente en los niños. Son ejemplos: 1) La población pediátrica susceptible de la resincronización cardiaca presenta complejo QRS ancho cuyo valor debe ajustarse a la edad y no a un valor de corte preestablecido por estudios multicéntricos en adultos (QRS > $150 \mathrm{mseg})$. 2) La población pediátrica con cardiomiopatía dilatada (CD) se beneficia de la resincronización cardiaca más temprana (CF I-II) pues en la clase funcional ıv la respuesta es más pobre que en los adultos. 3) La resincronización en la población pediátrica muestra resultados generales más efectivos y una respuesta favorable más temprana que en los adultos (77-89\% vs. $70 \%$ en adultos $)^{16,17}$. 4) La causa más frecuente de la terapia de la resincronización cardiaca en los niños es la cardiopatía dilatada secundaria a la estimulación ventricular derecha a diferencia de los adultos con cardiopatía dilatada secundaria a la enfermedad isquémica del corazón ${ }^{12,16}$.

\section{Conclusiones}

A pesar de los resultados positivos descritos y el continuo aumento en el número de los dispositivos cardiacos implantables en los niños, actualmente no existen estudios aleatorizados, prospectivos y/o controlados evaluando la resincronización cardiaca ni se cuenta con las guías para su implementación en la falla cardiaca pediátrica. La resincronización cardiaca biventricular transvenosa es técnicamente viable en los niños a diferentes edades, es factible actualizarla durante el crecimiento o según la necesidad del paciente, pero requiere una planeación y aproximación meticulosa especialmente el análisis de los vasos de acceso. Se requiere finalmente una mayor comprensión de la fisiopatología de la insuficiencia cardiaca, de la cardiopatía dilatada y de la disincronía electromecánica en la población pediátrica para definir y fomentar nuevas estrategias terapéuticas basadas en la terapia de la resincronización cardiaca.

\section{Responsabilidades éticas}

Protección de personas y animales. Los autores declaran que para esta investigación no se han realizado experimentos en seres humanos ni en animales.

Confidencialidad de los datos. Los autores declaran que en este artículo no aparecen datos de pacientes.

Derecho a la privacidad y consentimiento informado. Los autores han obtenido el consentimiento informado de los pacientes y/o sujetos referidos en el artículo. Este documento obra en poder del autor de correspondencia.

\section{Conflicto de intereses}

Los autores declaran no tener ningún conflicto de intereses.

\section{Bibliografía}

1. Webster G, Zhang JRD. Comparision of epidemiology and comorbidities of heart failure in the pediatric adult populations: a retrospective, cross-sectional study. BMC Cardiovasc Disord. 2006;6:23.

2. Janousek J, Tomek V, Chaloupecky VGR. Dilated cardiomyopathy associated with dual-chamber pacing in infants: improvement through either left ventricular cardiac resynchronization or programming the pacemaker off allowing intrinsic normal conduction. J Cardiovasc Electrophysiol. 2004;(15):470-4.

3. Chen CA, Chang Cl, Wang JK, Lin MT, Chiu SN, Chiu HH, et al. Restoration of cardiac function by setting the ventricular pacing at a lower range in an infant with congenital complete atrioventricular block and dilated cardiomyopathy. Int J Cardiol. 2008;(131):38-40. 
4. Yasuda K, Hayashi G, Ohuchi H, Ono Y, Yutani CES. Dilated cardiomyopathy after pacemaker implantation in complete heart block. Pediatr Int. 2005;(47):121-5.

5. Chen C-A, Wang J-K, Lin M-T, Lu C-W, Wu K-L, Chiu S-N, et al. Dilated Cardiomyopathy After Long-Term Right Ventricular Apical Pacing in Children With Complete Atrioventricular Block: Role of Setting of Ventricular Pacing. J Card Fail [Internet]. Elsevier Inc;. 2009;15(8):681-8. Available from: http: / / linkinghub. elsevier.com/retrieve/pii/S1071916409001146.

6. Tantengco MV, Thomas RLKP. Left ventricular dysfunction after long-term right ventricular apical pacing in the young. J Am Coll Cardiol. 2001;(37):2093-100.

7. Thambo JB, Bordachar P, Garrigue S, Lafitte S, Sanders P, Reuter $S$, et al. Detrimental ventricular remodeling in patients with congenital complete heart block and chronic right ventricular apical pacing. Circulation. 2004;(110):3766-72.

8. P.P. K. Chronic right ventricular pacing and cardiac performance: the pediatric perspective. Pacing Clin Electrophysiol. 2004;(27):844-9.

9. Molina JE. Surgical options for endocardial lead placement when upper veins are obstructed or nonusable. J Interv Card Electrophysiol [Internet]. 2004;11(2):149-54. Available from: http://www.ncbi.nlm.nih.gov/pubmed/15383779.

10. Bristow MR, Saxon LA, Boehmer J, Krueger S, Kass DA, DeMarco $\mathrm{T}$, et al. Cardiac resynchronization therapy with or without an implantable defibrillator in advanced chronic heart failure. $\mathrm{N}$ Engl J. Med. 2004; (350):2140-50.

11. Batra AS, Balaji S. Cardiac Resynchronization Therapy in Children. 2009; 5(1):40-4
12. Motonaga KS, Dubin AM. Cardiac resynchronization therapy for pediatric patients with heart failure and congenital heart disease: A reappraisal of results. Circulation. 2014;129(18): 1879-91.

13. Strieper M, Karpawich P, Frias P, Gooden K, Ketchum D, Fyfe $D$, et al. Initial experience with cardiac resynchronization therapy for ventricular dysfunction in young patients with surgically operated congenital heart disease. Am J Cardiol. 2004;94(10):1352-4.

14. Dubin AM, Janousek J, Rhee E, Strieper MJ, Cecchin F, Law IH, et al. Resynchronization therapy in pediatric and congenital heart disease patients: An international multicenter study. J Am Coll Cardiol. 2005;46(12):2277-83.

15. Moak JP, Hasbani K, Ramwell C, Freedenberg V, Berger JT, Dirusso G, et al. Dilated Cardiomyopathy Following Right Ventricular Pacing for AV Block in Young Patients: Resolution After Upgrading to Biventricular Pacing Systems. J Cardiovasc Electrophysiol [Internet]. 2006;17(10):1068-71. Available from: http://doi.wiley.com/10.1111/j.1540-8167.2006. 00565.x.

16. Janousek J, Gebauer RA. Cardiac resynchronization therapy in pediatric and congenital heart disease. Pacing Clin Electrophysiol. 2008;31 Suppl 1:S21-3. February.

17. Cecchin F, Frangini PA, Brown DW, Fynn-Thompson F, Alexander ME, Triedman JK, et al. Cardiac resynchronization therapy (and multisite pacing) in pediatrics and congenital heart disease: Five years experience in a single institution. J Cardiovasc Electrophysiol. 2009;20(1):58-65. 